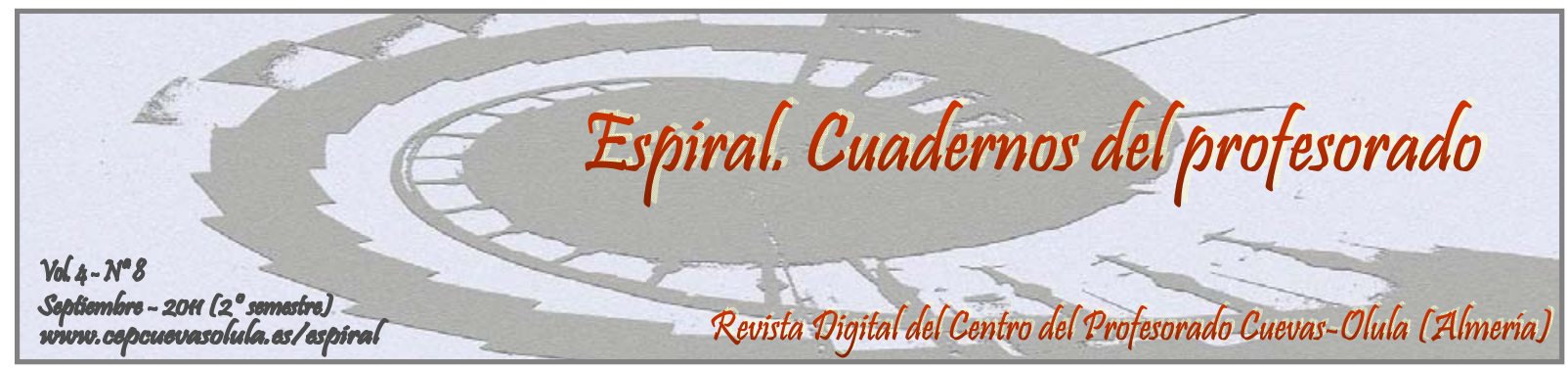

\title{
LAS ASIGNATURAS DE CIENCIAS EN LAS PRUEBAS DE ACCESO A LA UNIVERSIDAD: PERSPECTIVA DE GÉNERO
}

\author{
SUBJECTS OF SCIENCE IN TESTS OF UNIVERSITY ENTRANCE: GENDER
}

\author{
Francisco Manzano-Agugliaro, Josefina Martínez-García y Amós García-Cruz
}

\section{Escuela Superior de Ingeniería, Universidad de Almería, España}

RESUMEN: Tradicionalmente la elección de las opciones de ciencias en la etapa del bachillerato y luego la carrera universitaria ha estado marcada por estereotipos de género. En este trabajo se analizaron los resultados desde el enfoque de género de los resultados de las pruebas de acceso a la universidad de los bachilleratos de ciencias, en Almería, durante el período 2005-2010. Se trata de una muestra de 17.797 alumnos, repartidos en 8.531 chicos y 9.326 chicas. Las variables analizadas son: ratio de presentados, porcentaje de aprobados, calificaciones medias, decisión vocacional y distribución de las calificaciones según asignaturas. Los datos procesados analíticamente, permiten establecer comparaciones cualitativas entre ambos grupos, mostrándose las diferencias y contrastando resultados con la tendencia nacional. Se observa que, el porcentaje de aprobados y en las calificaciones medias, son favorables a los varones, aunque muy cerca de sus compañeras. Del estudio comparativo entre asignaturas se evidencian algunas descoordinaciones entre la formulación de la prueba de acceso a la universidad y la preparación para la misma desde el bachillerato. Gracias a este tipo de estudios es posible actuar en la mejora de programas educativos, tanto en igualdad de género como sobre las asignaturas que se alejan de la media del conjunto.

Palabras clave: Género, Prueba de Acceso a la Universidad, Ciencias, Bachillerato.

ABSTRACT: Traditionally the choice of science options at the stage of high school and then university studies has been marked by gender stereotypes. This paper discusses the results from the gender point of view of the results of entrance examinations to the university in the Bachelor's degree of Science in Almeria, during the period 2005-2010. We analyzed a sample of 17,797 students, 8531 men and 9326 women. The variables analyzed are: presented ratio, percentage of pass, average skills, vocational choice and distribution of ratings according to subjects. Analytically processed data to provide excellent qualitative comparisons between the two groups, showing the differences and contrasting results with the national trend. It is noted that the pass rate and the average scores are favorable to men, although very close to women. Comparative study between subjects are evident lack of coordination between the development some of the entrance examination to college and preparing for it since high school. With this type of study is possible to act on the improvement of educational programs, both gender equality and on subjects that are far from the average for all.

Key words: Gender, University Access Exams, Science studies, bachelor's degree. 
Manzano-Agugliaro, F., Martínez-García, J. y García-Cruz, A. (2011). Las asignaturas de ciencias en las pruebas de acceso a la universidad: perspectiva de género. Espiral. Cuadernos del Profesorado, 4(8), 3-12. Disponible en: http://www.cepcuevasolula.es/espiral.

Fecha de recepción: 23/06/2011

Fecha de aceptación: 05/08/2011
Enviar correspondencia a: fmanzano@ual.es

\section{1.- INTRODUCCIÓN}

\section{Tipos de bachillerato}

El bachillerato es la etapa posterior a la Educación Secundaria Obligatoria, pero a diferencia de ésta, tiene carácter voluntario y una duración de entre 2 y 4 años. Mediante la realización del Bachillerato el alumnado debe adquirir la suficiente madurez intelectual y humana, así como los conocimientos y habilidades para poder desenvolverse como ciudadanos competentes y responsables. Además posibilita el acceso a la Formación Profesional de Grado Superior y a los Estudios Universitarios previa prueba de acceso a la universidad (PAU). La estructura y enseñanzas mínimas del Bachillerato se regulan mediante Real Decreto 1467/2007. En Andalucía por el Decreto 416/2008 y la Orden 5 de Agosto de 2008, que regula su currículo.

Existen cuatro modalidades de Bachillerato, y dentro de cada una se plantean itinerarios diferentes que orientan al alumno hacia una especialización progresiva. Los tipos de Bachillerato son: Artes, Humanidades y Ciencias Sociales, Tecnológico y Ciencias de la Naturaleza y la Salud, (Consejería de Educación de la Junta de Andalucía, 2011). Este trabajo se centra en los dos últimos tipos de Bachillerato, considerando las asignaturas propias de los Itinerarios de Ciencias e Ingeniería, y de Ciencias de la Salud. La estructura de asignaturas se muestra de forma resumida en las tablas 1 y 2.

Tabla 1.- Bachillerato de Tecnología $(\mathrm{O}=$ Obligatoria, $\mathrm{E}=$ elegir 1$)$.

\begin{tabular}{|c|c|c|c|}
\hline Curso & Itinerario & Elección & Asignatura \\
\hline \multirow[t]{2}{*}{$1^{\circ}$} & \multirow[t]{2}{*}{ Único } & $\mathrm{O}$ & $\begin{array}{l}\text {-Física y Química } \\
\text {-Matemáticas I }\end{array}$ \\
\hline & & $\mathrm{E}$ & Dibujo Técnico I // Tecnología Industrial I \\
\hline \multirow{4}{*}{$2^{\circ}$} & \multirow{2}{*}{$\begin{array}{l}\text { Ciencia e } \\
\text { Ingeniería }\end{array}$} & $\mathrm{O}$ & $\begin{array}{l}\text { - Física } \\
\text { - Matemáticas II }\end{array}$ \\
\hline & & $\mathrm{E}$ & $\begin{array}{l}\text { Dibujo Técnico II // Tecnología Industrial I // } \\
\text { // Electrotecnia // Mecánica }\end{array}$ \\
\hline & \multirow{2}{*}{$\begin{array}{l}\text { Tecnología } \\
\text { Industrial }\end{array}$} & $\mathrm{O}$ & $\begin{array}{l}\text {-Electrotecnia } \\
\text {-Mecánica }\end{array}$ \\
\hline & & E & $\begin{array}{l}\text { Dibujo Técnico II // Física // } \\
\text { Matemáticas II // Tecnología Industrial I }\end{array}$ \\
\hline
\end{tabular}

\section{La Prueba de Acceso a la Universidad (PAU)}

La Prueba de Acceso a la Universidad (PAU) es un conjunto de exámenes acerca de las materias impartidas en $2^{\circ}$ de Bachillerato. La valoración conjunta de esta prueba y de los dos años de Bachillerato constata que el alumnado está suficientemente preparado para lograr el óptimo aprovechamiento de los estudios universitarios a los que optan. El requisito para realizar la prueba es el título de Bachiller y la prueba es válida para el acceso a todas las titulaciones de las universidades españolas.

La regulación de las Pruebas de Aptitud para el Acceso a la Universidad se remonta a la Ley 30/1974 de 24 de julio (BOE de 26 de julio) tras la que se articula la prueba mediante la Orden de 9 de enero de 1975, modificada por la Orden de 9 de octubre de 1979, (Muñoz-Repiso et al., 1991a). La configuración de la prueba ha sufrido continuas modificaciones hasta llegar a la actual PAU, puesta en 
marcha en el curso 2009-2010. Su marco normativo es el Real Decreto 1892/2008, de 14 de noviembre, (BOE del 24 de noviembre de 2008), en el que se regulan, a nivel estatal, las condiciones para el acceso a las enseñanzas universitarias oficiales de grado y los procedimientos de admisión a las universidades públicas españolas. Forman parte también de la normativa reguladora, la Corrección de errores del Real Decreto 1892/2008, de 14 de noviembre, publicada en el BOE del sábado, 28 de marzo de 2009 y las Órdenes EDU/1434/2009, de 29 de mayo, y EDU/3242/2010, de 9 de diciembre por las que se actualizan los anexos del Real Decreto 1892/2008, publicadas en los BOE $\mathrm{n}^{\circ} 135$, de 4 de junio de 2009 y 306, de 17 de diciembre de 2010.

Tabla 2.- Bachillerato de Ciencias de la Naturaleza y Ciencias de la Salud (O = Obligatoria, E = elegir 1).

\begin{tabular}{|c|c|c|c|}
\hline Curso & Itinerario & Elección & Asignatura \\
\hline \multirow[t]{2}{*}{$1^{\circ}$} & \multirow[t]{2}{*}{ Único } & $\mathrm{O}$ & $\begin{array}{l}\text {-Física y Química } \\
\text {-Matemáticas I }\end{array}$ \\
\hline & & $\mathrm{E}$ & Dibujo Técnico I // Biología y Geología I \\
\hline \multirow{4}{*}{$2^{\circ}$} & \multirow{2}{*}{$\begin{array}{l}\text { Ciencia e } \\
\text { Ingeniería }\end{array}$} & $\mathrm{O}$ & $\begin{array}{l}\text { - Física } \\
\text { - Matemáticas II }\end{array}$ \\
\hline & & $\mathrm{E}$ & $\begin{array}{l}\text { Dibujo Técnico II // Ciencias Tierra y } \\
\text { Medio Ambiente // Biología // Química }\end{array}$ \\
\hline & \multirow{2}{*}{$\begin{array}{l}\text { Tecnología } \\
\text { Industrial }\end{array}$} & $\mathrm{O}$ & $\begin{array}{l}\text { - Biología } \\
\text { - Química }\end{array}$ \\
\hline & & $\mathrm{E}$ & $\begin{array}{l}\text { Dibujo Técnico II // Física // Matemáticas } \\
\text { II // Ciencias Tierra y Medio Ambiente }\end{array}$ \\
\hline
\end{tabular}

El nuevo sistema para la PAU se compone de una fase general obligatoria y una fase específica voluntaria que permite subir nota. En cada ejercicio se ofrecen dos opciones a elegir por el alumno y la duración de cada examen es de una hora y media. En la fase general, se realizan cuatro exámenes ó cinco si existe lengua autonómica oficial: Lengua castellana y literatura, Lengua extranjera a elegir, Historia/Filosofía, y una materia de la modalidad de Bachillerato a elegir. El aprobado se obtendría con un 5, resultado del $60 \%$ de la nota media de Bachillerato y un $40 \%$ de la calificación de esta fase general (media aritmética de los ejercicios), con un mínimo de nota para dicha fase, de 4. La calificación obtenida tiene validez indefinida.

En la fase específica, los exámenes son sobre materias de modalidad y el alumno puede decidir el número de ejercicios a realizar, con un máximo de cuatro, aunque contarán las dos mejores. Es posible subir hasta cuatro puntos la nota de admisión ya que si la materia está adscrita a la rama de conocimiento del título a elegir, se pondera con un $20 \%$ en lugar de un $10 \%$. La caducidad de las notas de la fase específica es de dos años.

El alumno puede presentarse de forma ilimitada a la PAU, tanto a la fase general como a la específica, con el objeto de aprobar o también de subir nota. En este caso siempre se conservará la mejor calificación obtenida.

\section{Perfil de la Universidad Española en ciencias por género}

Actualmente, el Sistema Universitario español está constituido por 78 universidades: 50 públicas y 28 privadas, repartidas en 236 campus. En la Universidad española en el curso 2009-10 se matricularon 1.556.377 alumnos, de los cuales el 54,2\% eran mujeres. Este predominio se extiende a todos los niveles de formación universitarios (Ministerio de Educación, 2011).

El acceso igualitario de la mujer a la universidad española no se produjo hasta 1910, cuando el Conde de Romanones, Ministro de Instrucción Pública, firmó la Real Orden 8 de marzo de 1910 por la cual se concedía a la mujer la matriculación libre en la universidad en iguales condiciones que el hombre (Scanlon, 1987; Ballarín, 1989; Sánchez, 2010; García 2010). Antes de dicho año, las mujeres que deseaban cursar estudios universitarios precisaban de un permiso de la Dirección General de Instrucción Pública, que incluía un estudio previo particularizado que se dilataba en el tiempo, lo cual desalentaba a la mayoría de las aspirantes (Itatí, 2006). 
Según consta en el Instituto Nacional de Estadística, los primeros datos registrados por género sobre el Sistema Universitario Español datan de 1998. Sin embargo, con anterioridad se publicó en el BOE 21 marzo de 1984 la ratificación en España de la Convención sobre la Eliminación de todas Formas de Discriminación contra las Mujeres (CEDAW), de 1979 de la ONU. Por tanto, desde esa fecha, dicho tratado obligaba a España a estudiar estadísticamente a los estudiantes desagregados según género (De Foronda, 2010).

En lo que respecta a las pruebas de acceso a la universidad y según datos del último año disponible, 2009, dentro de los estudiantes matriculados se encuentran un 56,4\% de mujeres y un 43,6 $\%$ de varones. De estos, las mujeres aprobadas constituyen un $46,7 \%$ frente al $35,4 \%$ de hombres. La relación de mujeres matriculadas según ramas respecto al total del alumnado de cada rama, se mueve en una horquilla del 29,5\% para la rama Científico-Técnica y el 72,2\% de la rama Humanidades. Para la rama de Ciencias de la Salud el dato es del 66,5\% (Ministerio de Educación, 2011).

Por consiguiente, tanto los datos de los últimos años, como los de anteriores, confirman la tendencia de que las mujeres constituyen el género predominante entre los estudiantes universitarios españoles (Vázquez y Manassero, 2009) Dentro de las opciones de los bachilleratos de la rama de Ciencias, las mujeres siguen decantándose por la rama de Ciencias de la Salud. Existe una probabilidad mayor de que una mujer opte por esta opción, más típica de ellas, que por otras en las que además, son mayores sus tasas de abandono (Mau, 2003).

\section{Estudios de género en educación secundaria en España}

Estudios sobre la prueba de selectividad constatan que las mujeres tienen un mejor rendimiento académico en bachillerato, se presentan en mayor número a las pruebas de acceso, pero obtienen peores resultados en estos exámenes puntuales, como la prueba de acceso a la universidad, con excepción de la opción de Humanidades (Muñoz-Repiso et al., 1991b; Cuxart, 2000)

En estudios más recientes se concluye que existen estereotipos de género en el alumnado de la ESO en cuanto a materias, estudios y carreras, utilizando para la toma de decisiones vocacionales dichas percepciones diferenciadas (Manassero y Vázquez, 2003a, Martínez, Navarro y Yubero, 2009). En bachillerato, las mujeres que optan por las opciones de ciencias son minoritarias en la modalidad de tecnología, mientras que predominan en artes, sociales y humanidades (Vázquez y Manassero, 2009). Este hecho se atribuye a la mayor capacidad de entrega al prójimo que se le supone a la mujer (García, Padilla y Suárez, 2009) y a la percepción de que en los estudios técnicos están menos capacitadas que para otros en los que la memorización juega un papel más importante (Santana, Feliciano y Jiménez, en prensa). También juega un papel fundamental la tendencia androcéntrica apuntada por Manassero y Vázquez, en el área científica-técnica y las consecuencias negativas de su transmisión a las escuelas primarias y secundarias (Manassero y Vázquez, 2003b).

Además, según los resultados del informe del Ministerio de Educación respecto a los estudiantes universitarios del curso 2009, la rama de Ciencias de la Salud, es la única que experimenta un crecimiento sostenido del número de estudiantes. Por otro lado, la rama de Ciencias ha sufrido un 3,4\% de pérdida en el último curso. Estos datos nos ponen en alerta acerca de la necesidad de fomentar, sobretodo en la mujer, la importancia de su presencia en la actividad productiva asociada al ámbito científico-tecnológico (Ministerio de Educación, 2011). Esta actuación no debería limitarse únicamente al ámbito escolar, sino que debe extenderse también a la familia ya que ésta es un referente principal en la toma de decisiones académico-profesionales (Santana et al., 2011).

\section{2.- OBJETIVOS}

En este trabajo se planteó analizar los resultados académicos de la prueba de acceso a la universidad en asignaturas de ciencias, comparando los resultados entre ellas y desde la perspectiva de género, para comprobar si se cumplen los estereotipos de género en las asignaturas de ciencias. Para ello se analizaron por género: porcentaje de presentados a las pruebas, porcentaje de aprobados, porcentaje de elección de itinerarios, calificaciones medias por asignaturas, distribución de calificaciones por asignaturas. 


\section{3.- MATERIAL Y MÉTODOS}

Se han empleado los datos de los resultados de las pruebas de acceso a la universidad en el distrito de Almería, para la convocatoria de Junio, entre los años 2005 a 2010. Dichos datos mostraban información acerca del total de estudiantes y de mujeres, por lo que será necesario deducir los datos de los hombres. El estudio se restringió a las asignaturas mayoritarias de los itinerarios de los Bachilleratos de Ciencias: Biología, Ciencias de la Tierra y Medio Ambiente, Química, Matemáticas, Dibujo Técnico y Física. La población de estudio está formada por un total de 17.797 alumnos con la distribución por géneros reflejada en la tabla 3.

Para el reparto de asignaturas en los itinerarios se partió de la consideración de que son asignaturas propias del itinerario de Ciencias de la Salud: Biología - Ciencias de la Tierra y Medio Ambiente - Química, y propias del itinerario de Ciencias e Ingeniería las asignaturas: Matemáticas-Física y Dibujo

Tabla 3.- Porcentaje promedio de elección de itinerario por género en el período 2005-2010.

\begin{tabular}{ccc}
\hline & $\begin{array}{c}\text { Ciencias de la } \\
\text { Salud }\end{array}$ & $\begin{array}{c}\text { Ciencias e } \\
\text { Ingeniería }\end{array}$ \\
\hline Hombres & 39 & 61 \\
Mujeres & 65 & 35 \\
\hline
\end{tabular}
Técnico.

Para el estudio de la distribución de las calificaciones, se agruparonn por un lado, las calificaciones menores de 5 y el resto se mantuvieron según los rangos reflejados en los datos, entre: 5-6; 6-7; 7-8; 8-9 y 9-10.

\section{4.- RESULTADOS Y DISCUSIÓN}

\section{Elección de itinerarios del bachillerato de ciencias}

En la tabla 4, se refleja el porcentaje de hombres y mujeres que se examinaron de cada grupo de asignaturas, obteniéndose los promedios de todo el período 2005-2010. Se observa como existe un estereotipo de género, siendo para las mujeres el de Ciencias de la Salud y para los hombres el de Ciencias e Ingeniería, tal como ocurre en el resto de España y apuntan otros estudios e investigaciones (García et al., 2009, Vázquez y Manassero, 2009, Ministerio de Educación, 2011, Santana et al., en prensa).

Tabla 4.- Estadísticas de presentados y aprobados por género

( $\mathrm{n}=$ población estudiada, $\mathrm{P}=$ presentados, $\mathrm{A}$ = aprobados.

\begin{tabular}{ccccccc}
\hline \multirow{2}{*}{ Curso } & \multicolumn{3}{c}{ Hombres } & \multicolumn{3}{c}{ Mujeres } \\
\cline { 2 - 7 } & $\mathbf{n}$ & $\mathbf{\% ~ P}$ & $\mathbf{\% ~ A ~}$ & $\mathbf{n}$ & $\mathbf{\%} \mathbf{P}$ & $\mathbf{\% ~ A ~}$ \\
\hline 2005 & 1.498 & 48 & 68 & 1.621 & 52 & 62 \\
2006 & 1.283 & 47 & 71 & 1.433 & 53 & 62 \\
2007 & 1.335 & 46 & 69 & 1.537 & 54 & 65 \\
2008 & 1.498 & 47 & 60 & 1.661 & 53 & 62 \\
2009 & 1.518 & 48 & 67 & 1.647 & 52 & 67 \\
2010 & 1.399 & 50 & 72 & 1.427 & 50 & 66 \\
\hline Total/media & $\mathbf{8 . 5 3 1}$ & $\mathbf{4 8}$ & $\mathbf{6 8}$ & $\mathbf{9 . 3 2 6}$ & $\mathbf{5 2}$ & $\mathbf{6 4}$ \\
\hline
\end{tabular}

\section{Alumnado presentado y aprobado por género}

El reparto de porcentajes entre alumnas y alumnos respecto al total de presentados en el periodo estudiado, se refleja en la tabla 3. Según estos resultados se obtuvieron unos promedios del $48 \%$ de hombres frente al 52\% de mujeres, lo que quiere decir que se estudiaron más mujeres que hombres, en la población analizada. Estos datos siguen la tónica general de la Universidad española o incluso están más nivelados por género (43,6 \% de hombres y 56,4\% de mujeres).

En el 2010 se observó que aumentó el porcentaje de varones llegando a igualar al de mujeres. A fecha de hoy, este último dato no es posible contrastarlo con el nacional pero este resultado parece indicar la vuelta de los chicos a las aulas tras un período de bonanza económica en el que era más 
accesible el comienzo de la vida laboral y de la deseada independencia económica, después de la enseñanza obligatoria. Sin embargo, las mujeres se centraron más en la continuación de sus estudios de cara a una mayor preparación para enfrentarse a un competitivo mercado laboral.

En la tabla 3 se muestran los resultados de los porcentajes de hombres y mujeres aprobados respecto al total de su género. Se observó que los hombres tienen un mejor porcentaje de superación de la prueba, $68 \%$ para los hombres respecto al 64\% de las mujeres. La diferencia no fue apreciable, y está de acuerdo con los estudios que indican que los hombres realizan mejor los exámenes puntuales, como la prueba de acceso a la universidad, (Muñoz-Repiso et al., 1991b; Cuxart, 2000). Sin embargo a nivel nacional se observa una diferencia de aproximadamente diez puntos a favor de la mujer, al contrario que en nuestro estudio, donde la balanza se inclinó hacia los varones con excepción del año 2008 en el que las mujeres superaban a los hombres en un $2 \%$, y en 2009 con igualdad de porcentajes entre ambos.

\section{Calificaciones medias por asignaturas}

En este apartado se analizaron los datos relativos a las calificaciones medias de las asignaturas según género y se expresan los resultados medios del período 2005-2010, obteniéndose los resultados gráficos de la figura 1 . Se apreció que los hombres superaban a las mujeres en todas las asignaturas analizadas, con diferencias más acusadas en asignaturas como Física y Ciencias de la Tierra

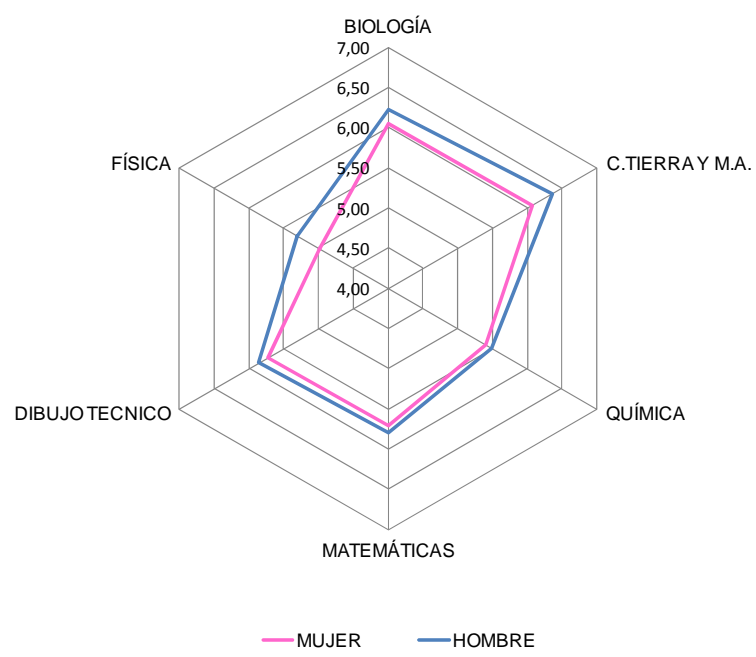

Figura 1.- Promedio de calificaciones medias según género y asignaturas en período 2005-2010.

- Medio Ambiente. Tal como ocurría con el porcentaje de aprobados, también ocurre con las calificaciones medias que los hombres realizan mejor los exámenes puntuales, como la prueba de acceso a la universidad, (Muñoz-Repiso et al., 1991b; Cuxart, 2000).

Si se analizan por asignaturas, a lo largo de los años, figura 2, se observa que estas tenían el mismo comportamiento, independientemente del género. Quizás la única que puede presentar diferencia es la de Ciencias de la tierra y Medio ambiente, favorable a los hombres.

Se observó como la Física, mantiene en general unos resultados muy bajos en ambos géneros, incluso por debajo del aprobado para las mujeres en 4 de los 6 años estudiados. En esta misma línea está la Química, que su tendencia es a una nota media de 5.5 a lo largo de los años, siendo especialmente mala este último año para las mujeres, configurándose como la de calificación más baja (la más difícil). En estas asignaturas, se podría deducir la necesidad de aumentar la coordinación entre los contenidos del bachillerato y la prueba de acceso para mejorar los resultados.

Por otro lado, hay asignaturas que mantuvieron un buen nivel de resultados para ambos sexos: la Biología y Ciencias de la tierra y Medio Ambiente. Esto puede indicar una buena coordinación entre los contenidos del bachillerato y la prueba de acceso. Como ya se ha mencionado respecto de las notas medias a lo largo de los años, en estas asignaturas, se podría deducir la necesidad de aumentar la coordinación entre los contenidos del bachillerato y la prueba de acceso para mejorar los resultados.

Llama la atención la variabilidad de la asignatura de Dibujo Técnico, la cual fluctúa hasta 3 puntos de media en años consecutivos. La tendencia en los tres últimos años ha sido mejorar la situación, llegando en el último año analizado a ser la que mejores resultados obtiene en ambos sexos.

La última convocatoria estudiada, 2010, ha sido la de mayor calificación media global y también la más igualada entre hombres y mujeres, lo que confirma que la situación económica actual fomenta en los estudiantes la motivación por obtener las calificaciones suficientes para entrar en la 
titulación deseada, esperando que le pueda asegurar su futuro laboral ante la gran competitividad existente.

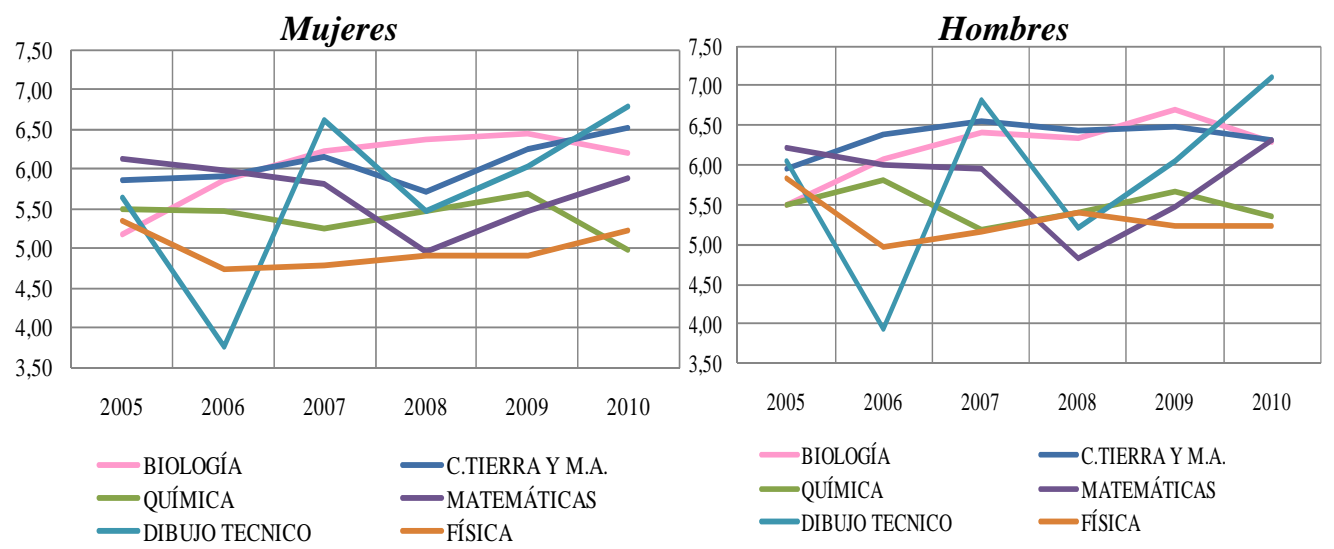

Figura 2.- Calificaciones medias por asignaturas según el género.

\section{Distribución de calificaciones por asignaturas.}

En la figura 3 se muestran, para cada asignatura, los promedios de los porcentajes de cada rango de calificación, respecto al total de cada género.

Para las asignaturas de Biología y Ciencias de la Tierra y Medio Ambiente los hombres obtuvieron las mejores calificaciones (8-9 y 9-10), en contra del estereotipo de género para estas asignaturas. Estas asignaturas también presentaron los porcentajes de fracaso más bajos para ambos géneros en el examen de la prueba de acceso, por debajo del $30 \%$.

La Química ofrece los mismos resultados en ambos géneros, destacando que más del $40 \%$ de los estudiantes no supera el examen de la prueba de acceso. Esto es más acusado todavía en la asignatura de Física con casi el $50 \%$ de los estudiantes.

Para las asignaturas de Matemáticas y Dibujo Técnico, con los datos analizados, no se cumple el estereotipo de género de clara ventaja en el resultado académico para los hombres, puesto que las mujeres tienen mayor porcentaje en las calificaciones entre 8-9 y los hombres entre 9-10. Estas asignaturas presentan los porcentajes de fracaso intermedios para ambos géneros en el examen de la prueba de acceso, por debajo del 35 \%. Respecto al Dibujo Técnico, y pese a la creencia generalizada de que los varones tienen mayores habilidades innatas por su mejor visión espacial, en este caso no son notables dichas diferencias.

El perfil del óptimo estudiante preuniversitario almeriense, según esto sería un chico con altas capacitaciones en Ciencias de la Tierra-Medio Ambiente y Biología, seguidas por el Dibujo Técnico y las Matemáticas. La Física y la Química serían las asignaturas en las que queda más desfavorecido.

La preuniversitaria almeriense seguiría una trayectoria sensiblemente paralela a la del hombre, algo por debajo en las calificaciones, especialmente en Física y muy igualada en Química, Matemáticas y Dibujo Técnico.

\section{5.- CONCLUSIONES}

Analizados los resultados de la prueba de acceso en asignaturas de ciencias en los últimos 6 años en el distrito de Almería, se observó una tasa de fracaso del 36 \% en general, pero mucho más acusado en dos asignaturas Física y Química, por encima del 40 \%. Se podría deducir la necesidad de aumentar la coordinación entre los contenidos del bachillerato y la prueba de acceso para mejorar los resultados de la prueba de acceso. 


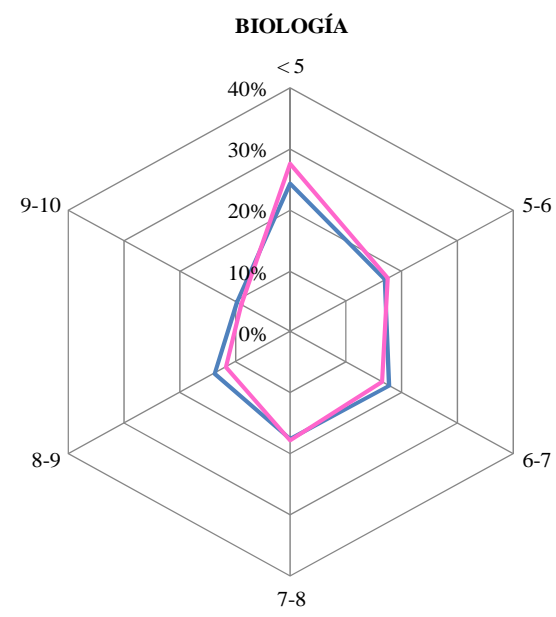

- HOMBRES — MUJERES

DIBUJO TÉCNICO

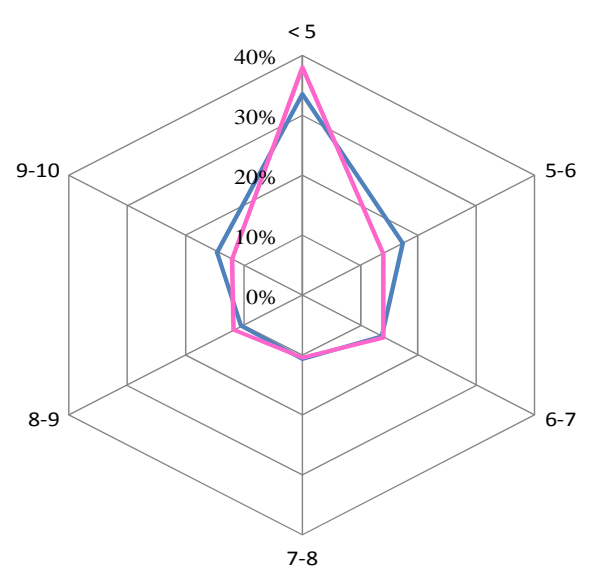

- HOMBRES — MUJERES

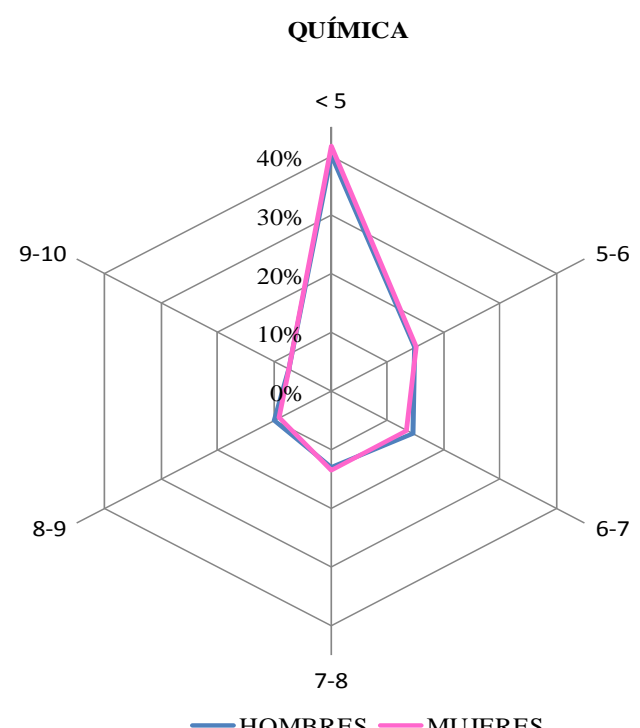

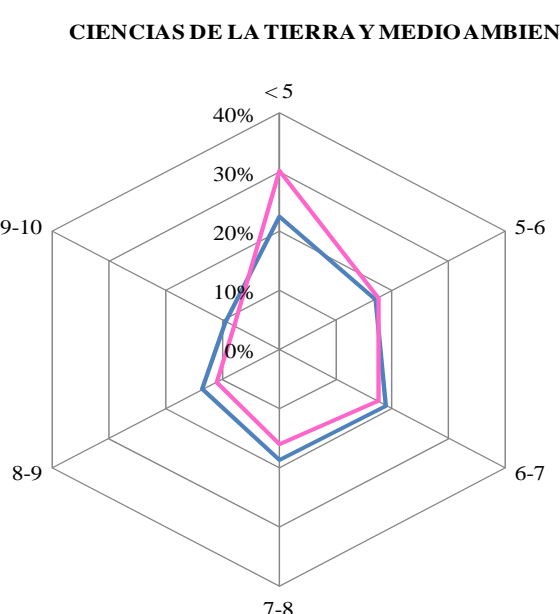

- HOMBRES —MUJERES

MATEMÁTICAS

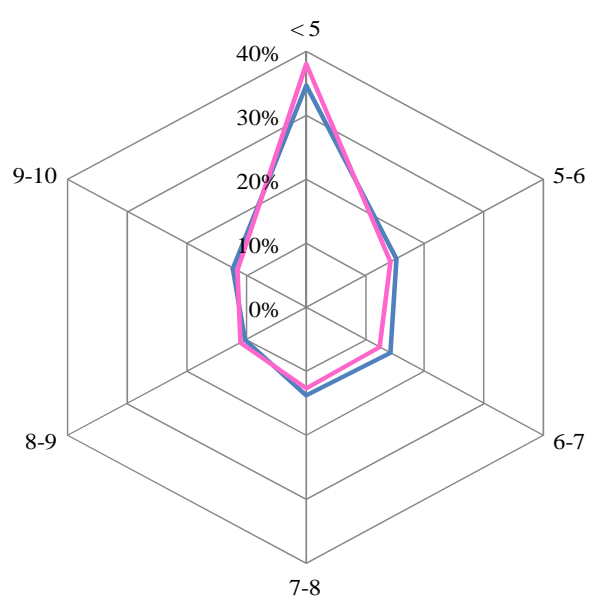

- HOMBRES — MUJERES

FÍSICA

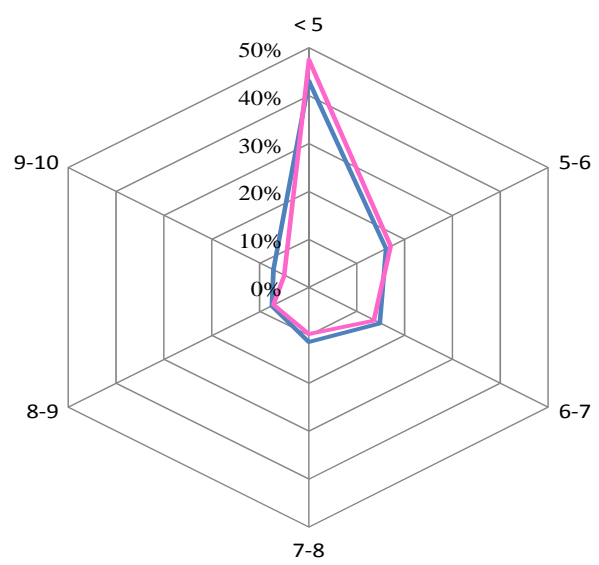

- HOMBRES — MUJERES

Figura 3.- Promedio de la distribución de calificaciones por asignaturas y género en el período 2005-2010. 
Respecto al género, se observa la misma tendencia que a nivel nacional de mayor porcentaje de presentados mujeres que hombres, pero en nuestro caso las diferencias entre ambos grupos son menores, y los hombres superan a las mujeres en el porcentaje de aprobados, al contrario de lo sucedido en el conjunto de España.

Se mantiene la polaridad existente en la elección de las opciones de ingeniería para los varones, y ciencias de la salud para mujeres. Sin embargo este hecho no está vinculado a la obtención de mejores resultados en dichas áreas ya que de media, los hombres superan a las mujeres en todas las asignaturas, pero más claramente en las asignaturas propias de Ciencias de la Salud: Biología y Ciencias de la Tierra. Por tanto el estereotipo de la elección de estudios en función del género no esta sustentado por los resultados de una prueba objetiva y simultanea como es la prueba de acceso a la Universidad.

Surge la reflexión de que las aptitudes del alumnado estudiado en este contexto particular, no se corresponde con sus elecciones vocacionales y con las percepciones sociales existentes que capacitan más a la mujer para el campo de ciencias de la salud y al hombre para las tareas científicotécnicas, ya que las diferencias entre ambos sexos no son apreciables. Por todo ello, se debe reflexionar sobre la conveniencia de trabajar en propuestas didácticas, acción tutorial a nivel de centro y de familia, para fomentar en el alumnado femenino la vocación científico-técnica, considerando por un lado, la gran importancia que ocupa esta actividad en la base productiva y por otro lado aumentar su presencia en las titulaciones que abastecen los puestos científico - técnicos en la sociedad.

\section{6.- REFERENCIAS BIBLIOGRÁFICAS}

Ballarín, P. (1989). La educación de la mujer española en el siglo XIX. Historia de la educación: Revista Interuniversitaria, 8, 245-260.

Cuxart, A. (2000). Modelos estadísticos y evaluación: tres estudios en educación. Revista de Educación, 323, 369-394.

De Foronda, P.V. (2010). Estadística universitaria desagregada por sexos, estado de la cuestión para la Asociación Clásicas y Modernas. Investigación para la Asociación Clásicas y Modernas [en línea], 1. Disponible en: http://www.ciudaddemujeres.com/articulos/IMG/pdf_Investigacion_CyM.pdf. [Consulta: 2011, 8 de junio]

García, S., Padilla, M.T. y Suárez, M., (2009). Los intereses académicos y profesionales de chicas que finalizan la escolaridad obligatoria. Revista de Educación, 349, 311-334.

García, M. (2010). La voz de las mujeres en la universidad. Revista de la Asociación de Sociología de la Educación, 3(3), 357-368.

Itatí, A. (2006). El acceso de las mujeres a la educación universitaria. Revista Argentina de Sociología, 4(7), 1146.

Manassero, M.A. y Vázquez, A. (2003a). Las mujeres científicas: un grupo invisible en los libros de texto. Investigación en la Escuela, 50, 31-45.

Manassero, M.A. y Vázquez, A. (2003b). Los estudios de género y la enseñanza de las ciencias. Revista de Educación, 330, 251-280.

Martínez, I., Navarro, R. y Yubero, S. (2009). Estereotipos de género entre los adolescentes españoles: imagen prototípica de hombres y mujeres e imagen de uno mismo. Información Psicológica, 95, 77-86.

Mau, W.C. (2003). Factors that influence persistence in science and engineering career aspirations. Career Development Quaterly, 51, 234-243.

Muñoz-Repiso Izaguirre, M., Muñoz Vitoria, F., Palacios Gómez,C., Valle López, J.M. y Escobar Fernández, M.C. (1991a). Introducción. En M. Muñoz-Repiso Izaguirre, F. Muñoz Vitoria, C. Palacios Gómez, J.M. Valle López y M.C. Escobar Fernández. Las calificaciones en las pruebas de aptitud para el acceso a la universidad. (pp. 7-21). Madrid: Centro de Publicaciones del Ministerio de Educación y Ciencia: C.I.D.E.

Muñoz-Repiso Izaguirre, M., Muñoz Vitoria, F., Palacios Gómez,C., Valle López, J.M. y Escobar Fernández, M.C. (1991b). Resultado según el sexo de los alumnos. En M. Muñoz-Repiso Izaguirre, F. Muñoz Vitoria, C. Palacios Gómez, J.M. Valle López y M.C. Escobar Fernández. Las calificaciones en las 
pruebas de aptitud para el acceso a la universidad. (pp. 103-115). Madrid: Centro de Publicaciones del Ministerio de Educación y Ciencia: C.I.D.E.

Sánchez J. (2010). De la casa al laboratorio: la mujer en la universidad española cumple su primer siglo. Uciencia, 3, 16.

Santana, L.E., Feliciano, L.A. y Jiménez, A.B. (en prensa). Toma de decisiones y género en el bachillerato. Revista de Educación, 359, 1-21.

Scanlon, G.M. (1987). La mujer y la instrucción pública: de la ley Moyano a la II ${ }^{\mathrm{a}}$ República. Historia de la Educación: Revista Interuniversitaria, 6, 193-208.

Vázquez, A. y Manassero, A. (2009). Patrones actitudinales de la vocación científica y tecnológica en chicas y chicos de secundaria. Revista Iberoamericana de Educación. 50(4), 1-11.

\section{Recursos en línea}

Consejería de Educación de la Junta de Andalucía (2011). [en línea]. Disponible en : http://www.juntadeandalucia.es/averroes/impe/web/contenido?pag=/contenidos/B/ApoyoAlCurriculo/C urriculoDeAndalucia/Seccion/Bachillerato/Planesestudio.Bachillerato. [Consulta 2011, 2 de junio]

Ministerio de Educación (2011). Datos y Cifras del Sistema Universitario Español. Curso 2010-11 [en línea] 423. Disponible en: http://www.educacion.gob.es/dctm/ministerio/educacion/universidades/estadisticasinformes/novedades/2011-datos-cifras-10-uv.pdf?documentId=0901e72b809384a4 [Consulta 2011, 6 de junio] 